\title{
ESTIMULANDO O APRENDIZADO NA ESCOLA BÁSICA POR MEIO DO PENSAMENTO COMPUTACIONAL, DA MATEMÁTICA E DA RESOLUÇÃO DE PROBLEMAS
}

\author{
Anelise Lemke Kologeski, Aline Silva De Bona, Natália Bernardo Nunes, Rafaela da Silva Bobsin, \\ Vithória da Silveira Batista e Vitória de Souza Fabricio \\ Instituto Federal de Educação, Ciência e Tecnologia do Rio Grande do Sul (IFRS) - Campus Osório \\ Rua Santos Dumont, 2127, Albatroz, Osório/RS, Brasil, 95520-000
}

\begin{abstract}
RESUMO
Este trabalho apresenta um projeto de extensão, com a finalidade de estimular o aprendizado dos participantes da Escola Básica através da inclusão digital, por meio da resolução de problemas que aliam a Matemática e o Pensamento Computacional, com a oferta de oficinas lúdicas. Os resultados mostram um rendimento satisfatório de até 37,87\% dos participantes, agregando na melhoria do Pensamento Computacional atrelado a resolução de problemas investigativos, intimamente relacionada com tarefas específicas da disciplina de Matemática, além de outras habilidades como raciocínio lógico e interpretação de texto, evidenciando a eficácia de criar-se novas práticas de ensino relacionadas à Tecnologia, visando melhorar o nível de aprendizagem dos alunos brasileiros.
\end{abstract}

\section{PALAVRAS-CHAVE}

Educação, Oficinas Lúdicas, Problemas Investigativos, Tecnologia

\section{INTRODUÇÃO}

Os meios em que vivemos estão em constante mudança, isso se deve muito a rápida evolução da tecnologia. Contudo, enquanto alguns tentam se adaptar para usufruir dela, outros já nascem tendo estas ferramentas digitais como comuns e adaptam-se com muito mais facilidade às mudanças, sendo estes os chamados "Nativos Digitais (ND)". De acordo com Palfrey e Gasser (2011), ND são aqueles nascidos após 1980, cujo contexto em que nasceram já trazia uma grande influência da internet e das tecnologias digitais. Eles apontam que a educação talvez não consiga acompanhar a evolução tecnológica, e mostram que muitos jovens não se interessam pelas aulas apenas expositivas, refletindo em índices educacionais. A Organização para a Cooperação e Desenvolvimento Econômico mostra em um ranking educacional de 36 países o Brasil como penúltimo (Veja, 2017). E, os últimos índices da Prova Brasil para os anos finais do Ensino Fundamental não apresentaram o resultado esperado no Brasil (IDEB, 2020). Para isso é necessária uma nova abordagem na educação, já que novas tecnologias devem ser usadas para novas práticas, uma vez que estamos caminhando em direção a um novo conceito de educação, conhecido como Educação 5.0, que consiste em um modelo educativo que integra além de conhecimento e tecnologia, associando os conceitos e habilidades cognitivas às competências socioemocionais, através da relação com um agente cosmopolita, desempenhado muitas vezes pelo professor (Morgado, 2016).

Contudo, em era digital, ainda existem muitas pessoas que não possuem fácil acesso a ferramentas digitais. De acordo com dados do Instituto Brasileiro de Geografia e Estatística (IBGE), publicado na revista Extra (Extra, 2018), cerca de 63 milhões de brasileiros não têm acesso à internet, e muitos nem sequer possuem interesse. Outra pesquisa aponta que cerca de 4,8 milhões de estudantes e crianças entre 9 e 17 anos não tem internet em suas casas (UICEF, 2020), confirmando a gravidade da situação. Levando em conta todas essas problemáticas pretende-se, através de um projeto de extensão atendendo alunos da Rede Pública de Ensino, proporcionar um momento de inclusão digital e de estímulo do aprendizado, utilizando atividades lúdicas como a problemas, envolvendo a Matemática e o Pensamento Computacional para que, por meio 
dessas atividades, os alunos fiquem instigados a participar e, com a troca e compartilhamento de saberes, tenham um momento de desenvolvimento, utilizando-se de ferramentas digitais.

Este trabalho está organizado da seguinte maneira: no Tópico 2 explicamos o porquê da escolha do Pensamento Computacional, enquanto que no Tópico 3 essa explicação é para a escolha do uso da Matemática. No tópico 4, apresentamos o porquê da abordagem de resolução de problemas, e o Tópico 5 traz a metodologia, bem como alguns dos problemas utilizados nas oficinas. O Tópico 6 trata da discussão sobre os resultados obtidos, e no Tópico 7 trazemos as conclusões e considerações finais deste trabalho.

\section{POR QUE O PENSAMENTO COMPUTACIONAL (PC)?}

O Pensamento Computacional (PC) está ligado com muitas tarefas do cotidiano, e não apenas ao uso de um computador. Programar um dispositivo GPS ou um despertador exige uma certa abstração do usuário, que instintivamente utiliza o PC para executar estas ações, por exemplo. Desta forma, o PC torna-se um requisito essencial para a vivência em sociedade nos dias de hoje, além de ser uma habilidade fundamental utilizada como critério de seleção por uma vaga no mercado de trabalho.

Para Wing (2010), o PC é compreendido como o método, a maneira, e a forma de pensar presente na abstração/lógica de um problema, bem como suas possíveis resoluções de modo que estas sejam representadas de uma forma que pode ser testada, ancorada em artefatos computacionais. Desta forma, o desenvolvimento de tarefas relacionadas com a programação básica pode ser um estímulo ao desenvolvimento do raciocínio dos alunos pois, ao desenvolvermos novas metodologias e estratégias utilizando novas ferramentas digitais, possibilitamos aos alunos a oportunidade de participação na construção de novos meios de educação, mais contextualizados com a atualidade, proporcionando também o desenvolvimento da autonomia que vem atrelado ao uso da tecnologia, proporcionando também a inclusão digital em muitas comunidades que não possuem fácil acesso a tecnologia.

\section{POR QUE A MATEMÁTICA?}

A Matemática é uma ciência, um saber que estabelece relação com a vida cotidiana. Compreender sua lógica proporciona ao estudante abstrair relações, construir fatos, estabelecer representações que ajudam seu poder de decisão e escolha em diferentes situações cotidianas e profissionais. De acordo com os Parâmetros Curriculares Nacionais (PCN), a Matemática tem essas finalidades e sofre um redirecionamento direto de acordo com o impacto das máquinas e tecnologias na vida das pessoas, pelo simples fato de se fazer necessário um conjunto de sistematizações e lógicas que nem sempre são necessárias sem as máquinas (Brasil, 1999). No entanto, saber Matemática não é saber apenas o algoritmo, mas compreender sua finalidade, interpretar seu resultado e conhecer sua construção, desde sua história até sua aplicabilidade, como por exemplo, o algoritmo da divisão. Assim, o PC pode ser explorado através de situações que envolvam a Matemática, sejam problemas investigativos, ou simples paralelos estabelecidos em situações como cita o trabalho de Silveira e Barcelos (2012). No entanto, os problemas investigativos são mobilizadores ao processo de aprendizagem dos estudantes, proporcionando autonomia, valorização dos seus saberes e viabilizando uma aprendizagem coletiva.

\section{POR QUE A RESOLUÇÃO DE PROBLEMAS?}

Cada vez mais o conhecimento em Computação torna-se um requisito aplicado nas mais diversas tarefas do cotidiano. Por isso, a oferta de oficinas com a oportunidade de inclusão digital se torna essencial para os estudantes quando trata-se de qualidade educacional, especialmente com o uso da resolução de problemas. Além de haver o estímulo ao PC, também estimula-se o raciocínio dos alunos através da Matemática, contribuindo para o desenvolvimento da autonomia, colaboratividade e aprendizado dos participantes. Por isso, optou-se pela atividades no formato de problemas investigativos. Para Ponte, Brocardo e Oliveira (2006), a ação de investigar é procurar conhecer o que não se sabe, descobrindo relações entre objetos 
Matemáticos conhecidos ou desconhecidos de maneira a procurar identificar suas propriedades e o processo de criação, contemplando os 4 passos, que podem ser identificados nos problemas propostos: Identificar o problema a resolver; Formular hipóteses; Realizar testes; Argumentar/validar, seja só ou em grupo.

As habilidades do PC consideradas neste trabalho foram guiadas pelo Currículo de Referência em Tecnologia e Computação do Centro de Inovação para a Educação Brasileira (CIEB, 2020), que tem como principal objetivo apoiar redes de ensino e escolas para inclusão de temas relacionados com a Tecnologia e Computação em suas propostas curriculares. Desta forma, as atividades desenvolvidas na perspectiva do PC podem contribuir para a construção do pensamento lógico e para o desenvolvimento do raciocínio por meio dos quatro pilares básicos do Pensamento Computacional, que são: decomposição, reconhecimento de padrões, abstração de um problema e algoritmos (Brackmann, 2017).

Já no aspecto matemático, existe a questão da interpretação e da identificação, por muitas vezes, do uso de uma ou mais variáveis, que podem quantificar a quantidade de passos e instruções que serão realizadas dentro de um algoritmo. Além disso, o teste para verificação dos valores equivalentes pode ser realizado em tempo real, na própria plataforma adotada, estimulando os estudantes a testarem suas hipóteses durante a execução da oficina, para que possam provar a eficácia de suas escolhas.

Através da resolução de problemas, especialmente envolvendo a Matemática, é possível associar o PC na Escola Básica, segundo Silveira e Barcelos (2012), proporcionando aos estudantes um desenvolver habilidades e competências comuns e complementares da área da Matemática e da Informática. Com isso, o ensino de Matemática pode explorar uma tendência da resolução de problemas, contemplando o PC, e viabilizando um processo de abstração mais complexo na Escola Básica de forma atrativa aos estudantes.

\section{METODOLOGIA}

Alguns países já estão incluindo disciplinas de Robótica e Programação em sala de aula como componentes do currículo escolar, pois acreditam no caráter interdisciplinar que o PC apresenta. Dentre eles, o que mais se destaca é o Reino Unido, onde em 2013 o Governo do país decidiu reformular o modelo antigo de aprendizagem, que era voltado apenas para conhecimentos básicos de Informática (como planilhas e editores de texto) para um currículo voltado à área da Computação e ao desenvolvimento de Algoritmos, tornando obrigatória a Programação nas escolas (Gazeta, 2016). Por outro lado, no Brasil, a Base Nacional Comum Curricular (BNCC, 2020) ainda não efetivou nenhuma integração entre conceitos da Informática e as matérias básicas do currículo escolar, o que evidencia a necessidade de iniciativas externas e, por isso, diversos trabalhos apresentam propostas semelhantes ao nosso projeto, como Neto (2016) e Garlet et al. (2016), que fazem uso de recursos digitais focando na educação infantil. Contudo, nosso projeto se diferencia por atender alunos de $9^{\circ}$ ano do Ensino Fundamental, permitindo ao professor participar das atividades, atendendo uma região geográfica específica e disponibilizando os passos das oficinas tanto com o uso de recursos digitais quanto de computação desplugada, se assim a escola desejar, permitindo que as escolas sejam atendidas mesmo sem a disponibilidade de computadores. Todas as atividades envolvem a capacidade de interpretação de texto do estudante, bem como o raciocínio lógico, para que o aluno consiga atingir os objetivos propostos no enunciado de cada atividade. Cada participante realiza as atividades propostas em seu próprio tempo. Para auxiliar os alunos que possuem menos facilidade, os bolsistas estão sempre disponíveis durante as oficinas, tirando dúvidas e prestando o auxílio necessário. As oficinas foram planejadas para a duração máxima de 4 horas, e elas podem ser realizadas na própria escola, ou no IFRS Campus Osório, de acordo com a decisão da escola convidada, diante da necessidade de recursos disponíveis ou de transporte para se deslocarem até o IFRS. Cada oficina é sempre acompanhada pela orientadora do projeto e pelo(a) professor(a) responsável pela turma.

Assim, através da realização das oficinas, os alunos aprendem noções relacionadas com a tecnologia e com o uso de comandos que envolvem a programação básica, por meio de atividades lúdicas, procurando instigá-los a conhecerem mais sobre os recursos digitais, bem como buscando um aprendizado significativo e efetivo que pode ser aplicado nas tarefas do cotidiano, assim como estimulando a capacidade de resolver os problemas do cotidiano, desenvolvendo múltiplas soluções, bem organizadas, com uma sequência de passos bem definidos e organizados. 
A metodologia desenvolvida permite relacionar diferentes conteúdos juntamente com as atividades aplicadas, envolvendo principalmente noções matemáticas, interpretação de texto, raciocínio lógico e o PC, favorecendo uma educação integral, como defende a Base Nacional Comum Curricular (BNCC, 2020). Os processos de aprendizagem envolvidos são potencialmente ricos para o desenvolvimento de competências fundamentais para o letramento matemático (raciocínio, representação, comunicação e argumentação) e para o desenvolvimento do PC. Neste contexto, as atividades proporcionadas visam aplicar os conceitos básicos da Computação no dia a dia dos estudantes, fazendo uso de temas, personagens, animações e conteúdos da cultura pop e adolescente, amplamente conhecidos pelos alunos participantes das oficinas. Para isso, optou-se por utilizar recursos de forma gratuita na plataforma digital Code.org, que é mundialmente reconhecida.

Para promover a inclusão digital aplicando-se as atividades propostas, as escolas podem escolher utilizar o laboratório de informática na própria escola, ou realizar o deslocamento até a nossa instituição de ensino. Para o desenvolvimento das atividades, o principal recurso necessário é o acesso à Internet através dos computadores, e o suporte da equipe de execução do projeto para orientação das tarefas. As oficinas são de até 4 horas, conforme disponibilidade dos participantes, e normalmente as tarefas são realizadas aos pares.

Alguns jogos da plataforma Code.org são apresentados aos estudantes durante as oficinas, e para a conclusão das tarefas os alunos precisam ordenar os comandos disponibilizados, em blocos, arrastando-os e criando pequenos algoritmos de execução, que devem ser testados para verificação e seguimento das atividades. Neste trabalho decidimos apresentar os 5 problemas que norteiam as tarefas, dentre todas as atividades realizadas. Estes problemas são apresentados no início da oficina, permitindo avaliar o conhecimento prévio dos estudantes, e novamente após a oficina, permitindo assim avaliar se o aluno conseguiu desenvolver melhor o seu raciocínio e sua compreensão em torno das tarefas propostas, possibilitando uma comparação entre os resultados obtidos antes e depois das oficinas.

\subsection{Problema 1}

O Problema 1 consta na Figura 1, e exige que o aluno reflita sobre o posicionamento do personagem que irá se movimentar, bem como a quantidade de passos que será necessária para a conclusão da atividade. Além das noções de espaçamento, como direita e esquerda, o aluno deverá identificar a correta ordem dos comandos apresentados, bem como a distância que será percorrida pelo personagem. Para isso, ele precisa compreender o cenário e o objetivo, sendo conduzido e auxiliado pela equipe de execução do projeto.

Problema 1. Observe o cenário onde o pássaro Red deseja chegar no porquinho. É correto afirmar que a sequência de comandos que permite o Red atingir o porquinho é:
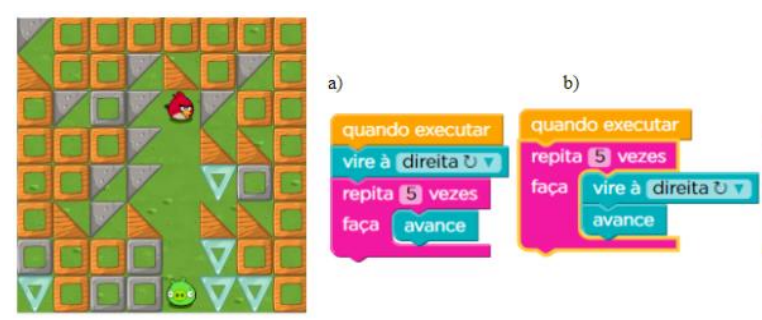
c)

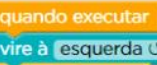
vire à esquerda
repita 5 vezes faça avance

Figura 1. Problema 1, baseado no jogo "Labirinto Clássico" da plataforma Code.org

\subsection{Problema 2}

O Problema 2 traz um cenário onde uma abelha precisa coletar duas vezes o néctar de duas flores, como pode ser visualizado na Figura 2. Para isso, o aluno já recebe de forma ordenada os comandos que serão utilizados, e ele apenas precisa interpretar e identificar qual é o valor que satisfaz a quantidade de repetições necessárias para a conclusão da tarefa. 
Problema 2. A abelhinha deseja coletar duas vezes o néctar das duas flores, conforme a figura ao lado.

Para isso, ela utilizará uma sequência de ações, conforme o código apresentado. Para que ela consiga coletar todos os néctares, qual o número que deve ser substituído pelo 0 no bloco rosa (repita __ vezes)?
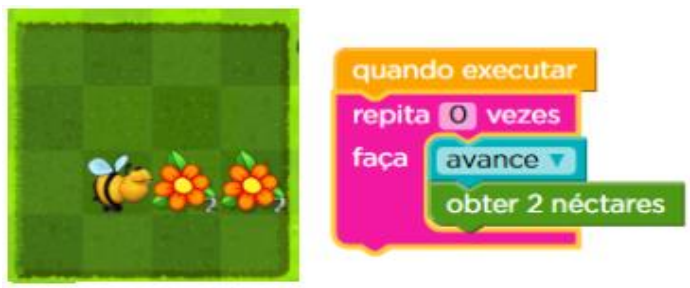

$\begin{array}{llll}\text { a) } 1 & \text { b) } 2 & \text { c) } 4\end{array}$

Figura 2. Problema 2, baseado no jogo "Abelha: Sequência" da plataforma Code.org

\subsection{Problema 3}

O Problema 3 oferece opções prontas de algoritmos onde o estudante deve identificar a correta movimentação do personagem Zumbi, avaliando se essa movimentação deve ocorrer para a direita ou para a esquerda em cada circunstância, como pode ser visualizado na Figura 3. Na atividade, não há uso de variáveis diretamente, como acontece nos demais problemas. Contudo, o aluno deve interpretar o posicionamento do personagem para conseguir obter a sequência correta da movimentação. E desse problema se propõe outros na sequência cada um agregando um ou outro elemento lógico para o pensamento computacional e para a resolução de um problema investigativo de matemática.

Problema 3. O zumbi deve chegar até a flor sorridente (semelhante a um girassol). Quais das opções mostra o conjunto de instruções corretas para que ele chegue até a flor?
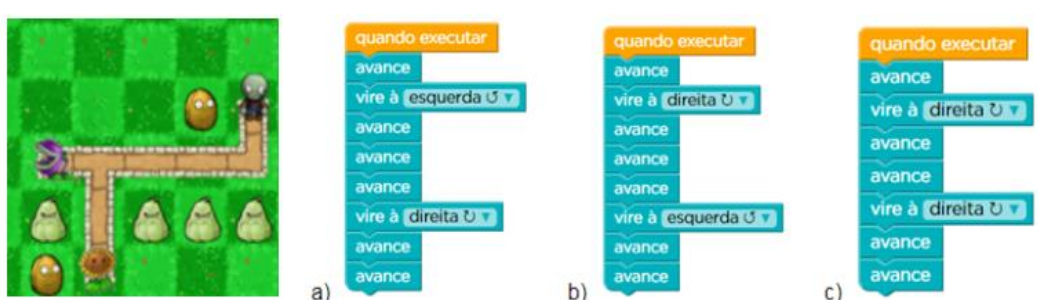

Figura 3. Problema 3, baseado no jogo "Labirinto Clássico" da plataforma Code.org

\subsection{Problema 4}

O Problema 4 também exige que o aluno seja capaz de abstrair a quantidade de movimentos através do comando "repetir até", e construa uma ideia de generalização do processo de resolução nesse bloco de atividades, como pode ser visualizado na Figura 4.

Problema 4. Levando em consideração a imagem ao lado, qual bloco de comando que está faltando e deve ser introduzido na imagem do centro para atingir o girassol?

a) Avance. b) Vire à direita. c) Vire à esquerda.
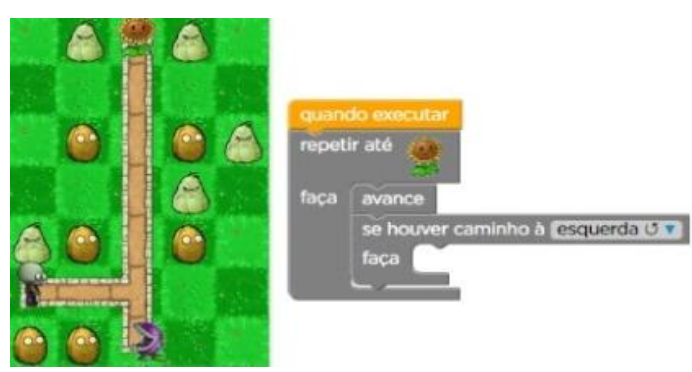

Figura 4. Problema 4, baseado no jogo "Labirinto Clássico" da plataforma Code.org 


\subsection{Problema 5}

O Problema 5 oferece opções que devem ser interpretadas e combinadas pelos estudantes, apresentando uma maior complexidade de interpretação, e apropriação do pensamento lógico atrelado ao computacional,, como pode ser visualizado na Figura 5.

Problema 5. Observe a situação em que se encontra a Abelha no Quadro 1 e compare com os comandos do Quadro 2. Levando em conta que a abelha precisa tirar o néctar de todas as flores do Quadro 1, a partir da sequência de comandos apresentada no quadro 2, diga se as sentenças abaixo são verdadeiras ou falsas.

( ) A abelha conseguirá, utilizando os comandos do Quadro 2, coletar todo o néctar do Quadro 1.

( ) A abelha não conseguirá, utilizando os comandos do Quadro 2, coletar todo o néctar do Quadro 1.

( ) Alterando o "repita 2 vezes" para "repita 3 vezes" do Quadro

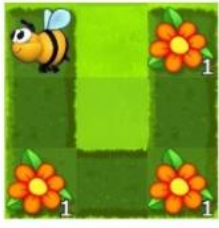

Quadro 1

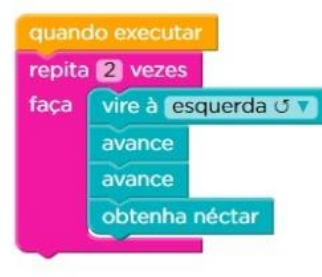

Quadro 2

2, a abelha conseguirá coletar todo o néctar do Quadro 1.

( ) Alterando o "repita 2 vezes" para "repita 1 vez", a abelha conseguirá coletar todo o néctar do Quadro 1.

() A abelha não conseguirá coletar todo o néctar do tabuleiro se alterarmos o "repita 2 vezes" para "repita 3 vezes".
a) $\quad V, F, F, V, V$.
b) $F, V, V, F, F$.
c) $F, F, V, F, F$.

Figura 5. Problema 5, baseado no jogo "Abelha: Sequência” da plataforma Code.org

\subsection{Requisitos e Habilidades Necessárias para a Resolução dos Problemas}

Diante dos problemas apresentados, foram listados na Tabela 1 aqueles requisitos considerados necessários para a devida interpretação e realização de cada um deles, em acordo com as habilidades listadas pelo (CIEB, 2020) e por (Brackmann, 2017), bem como por Ponte, Brocardo e Oliveira (2006), como já mencionado anteriormente no Tópico 4.

Tabela 1. Requisitos de cada problema

\begin{tabular}{|c|c|c|}
\hline Problemas & Habilidades do Pensamento Computacional & Habilidades da Matemática \\
\hline 1 & $\begin{array}{l}\text { Reconhecimento de padrões (passos de deslocamento e } \\
\text { direções), decomposição (múltiplas instruções), algoritmos } \\
\text { (ordem das instruções) e abstração (associar a Matemática } \\
\text { ao fator multiplicativo da repetição). }\end{array}$ & $\begin{array}{l}\text { Identificar a quantidade e ordenação de } \\
\text { instruções necessárias, e a repetição de } \\
\text { passos (valor da variável). }\end{array}$ \\
\hline 2 & $\begin{array}{l}\text { Reconhecimento de padrões (passos de deslocamento e } \\
\text { direções), decomposição (múltiplas instruções), algoritmos } \\
\text { (ordem das instruções) e abstração (associar a Matemática } \\
\text { ao fator multiplicativo da repetição). }\end{array}$ & $\begin{array}{l}\text { Identificar a quantidade de repetições } \\
\text { necessárias para a conclusão da tarefa (valor } \\
\text { da variável). }\end{array}$ \\
\hline 3 & $\begin{array}{l}\text { Reconhecimento de padrões (passos de deslocamento e } \\
\text { direções), decomposição (múltiplas instruções), algoritmos } \\
\text { (ordem das instruçôes) e abstração (associar a Matemática } \\
\text { com a quantidade de instruções necessárias). }\end{array}$ & $\begin{array}{l}\text { Identificar a quantidade e ordenação das } \\
\text { instruções necessárias. }\end{array}$ \\
\hline 4 & $\begin{array}{l}\text { Reconhecimento de padrões (passos de deslocamento e } \\
\text { direções), decomposição (múltiplas instruções), algoritmos } \\
\text { (ordem das instruções) e abstração (associar instruções } \\
\text { sem saber a quantidade real de passos necessários). }\end{array}$ & $\begin{array}{l}\text { Abstrair a quantidade exata de passos } \\
\text { necessários para a conclusão do objetivo. }\end{array}$ \\
\hline 5 & $\begin{array}{l}\text { Reconhecimento de padrões (passos de deslocamento e } \\
\text { direções), decomposição (múltiplas instruções), algoritmos } \\
\text { (ordem das instruções) e abstração (associar a Matemática } \\
\text { ao fator multiplicativo da repetição). }\end{array}$ & $\begin{array}{l}\text { Identificar a quantidade de instruções } \\
\text { necessárias e a repetição de passos. }\end{array}$ \\
\hline
\end{tabular}




\section{RESULTADOS E DISCUSSÃO}

Em 2019, 169 alunos foram atendidos, de 8 instituições distintas. Diversas atividades foram abordadas durante as oficinas, mas apenas os 5 problemas apresentados é foram utilizados para comparar o aprendizado dos alunos antes e após a realização das tarefas propostas, estes resultados podem ser visualizados no gráfico da Figura 6, que traz o percentual de acertos por problema no teste realizado no início da oficina (cinza claro) e o percentual de acertos por problema no fim das oficinas (preto). É possível observar que a uma variação no percentual, que indica uma melhoria, sendo essa de 19,52\% para o Problema 3 até 37,87\% para o Problema 4, confirmando que houve um desenvolvimento dos participantes com a realização das atividades.

A Figura 7, é referente a quantidade de problemas resolvidos corretamente por aluno nos testes realizados antes e após o início das atividades. É possível observar que quantidade de alunos que acertaram apenas entre 1 e 3 questões inicialmente é bem grande (81\%), porém é visível que após as atividades realizadas a quantidade de alunos que acertaram entre 3 e 5 problemas aumentou consideravelmente, representando $87 \%$ dos participantes. Isso significa uma melhoria na compreensão e resolução das atividades propostas, fazendo com que os estudantes passassem a acertar um maior número de questões no teste final.

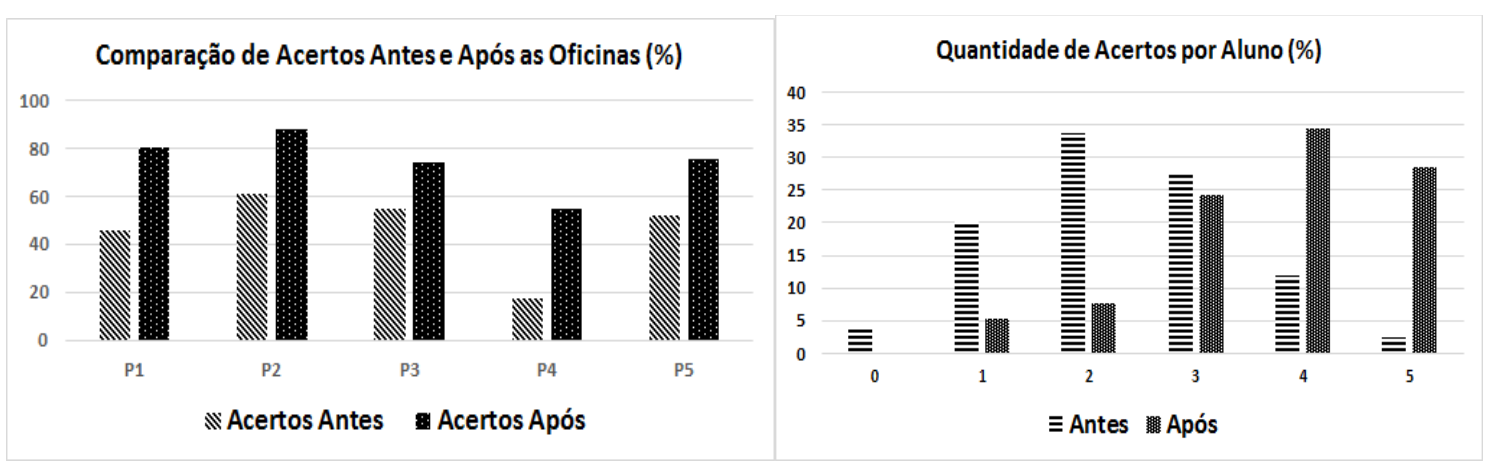

Figura 6. Quantidade de acertos (\%) por problema antes e após a realização das oficinas

Figura 7. Quantidade de acertos (\%) por aluno antes e após a realização das oficina

\section{CONCLUSÃO E CONSIDERAÇÕES FINAIS}

Atualmente, cada vez mais vivemos e temos de nos adaptar a processos que parecem simples mas que não são, como por exemplo, se inscrever num evento online: ler as orientações, analisar se está de acordo com o que procura-se, fazer a inscrição, pagar a inscrição, organizar o trabalho a submeter, aguardar a avaliação, enviar novamente com os nomes, e organizar a apresentação. A descrição sequencial parece simples, mas não é de fato, pois quer um processo de abstração conceitual, porque se faz uso de mais de uma tecnologia e também exige-se uma compreensão de verificação. Tal processo se assemelha a resolução de um problema investigativo, como os problemas apresentados neste trabalho, não existindo uma regra de ordem rígida, mas sim uma sistematização e autonomia fundamental. Esse processo está intimamente associado a compreensão ou aplicação, por exemplo, de um conceito de Matemática, como ilustra-se: para marcar três pontos num plano cartesiano e determinar a área do seu triângulo, sem usar fórmula, primeiro precisamos saber marcar os pontos, avaliar o tipo de triângulo para ver qual a melhor forma de calcular sua área, depois encontrar os elementos para o cálculo, e encontrar o valor da área para avaliar se está correto com o que se apresenta.

Diante desse contexto através da disciplina de Matemática da Escola Básica, encontra-se um espaço para proporcionar aos estudantes o início do processo do Pensamento Computacional, que é tão importante para realizar muitas atividades da vida cotidiana, de outras áreas do conhecimento e até posteriormente em escolhas profissionais. Em tempos atuais, o período presencial da escola já não é mais suficiente, e por isso vem se construindo a ideia de que é necessário desde os primórdios estudar em casa, com certa autonomia. Diante deste tempo de incertezas, perante a pandemia do novo Coronavírus, esse processo de autonomia e de estudo em casa se acentuou, se fazendo necessária a oportunização de alternativas ao ensino clássico e tradicional, evidenciando projetos de extensão como as oficinas aqui apresentadas, onde há um trabalho de readaptação e reconstrução para que sejam ministradas e propostas para uso remoto, em casa, através de ambientes online, requerendo muitas adaptações e aprendizagens por todos envolvidos. 


\section{AGRADECIMENTOS}

Agradecemos ao Instituto Federal de Educação, Ciência e Tecnologia do Rio Grande do Sul pela oportunidade de realizar esse projeto e que por meio do Edital IFRS nº 71/2019 financiou a publicação do artigo e a participação de uma autora no evento. Por fim agradecemos a todos os que tornaram possível as parcerias do projeto com as escolas da região, para a realização das oficinas.

\section{REFERÊNCIAS}

Barcelos, T.; Silveira, I., 2012. Pensamento Computacional e Educação Matemática: Relações para o Ensino de Computação na Educação Básica. XX Workshop sobre Educação em Computação (WEI), Curitiba/Brasil, 2012.

Base Nacional Comum Curricular (BNCC), 2020. Disponível em: http://basenacionalcomum.mec.gov.br/wpcontent/uploads/2018/12/BNCC_19dez2018_site.pdf. Acesso em: 24 jul. 20.

Brackmann, Christian. Desenvolvimento do Pensamento Computacional Através de Atividades Desplugadas na Educação Básica. Tese (Doutorado). Universidade Federal do Rio Grande do Sul (UFRGS), Porto Alegre, RS, Brasil, 2017. Disponível em: https://lume.ufrgs.br/handle/10183/172208. Acesso em: 11 fev. 20.

Brasil, 1999. Ministério da Educação; Secretaria de Educação Básica; Conselho Nacional de Educação. Parâmetros Nacionais da Educação: Ensino Médio. Brasília: MEC; SEMTEC; 4v.

CIEB, 2020. Centro de Inovação para a Educação Brasileira. Disponível em: https://curriculo.cieb.net.br/. Acesso em: 25 jul. 20.

Extra, 2018. IBGE: 63 milhões de brasileiros não usam a internet. Muitos sequer têm interesse. 2018. Disponível em: $<$ https://extra.globo.com/noticias/economia/ibge-63-milhoes-de-brasileiros-nao-usam-internet-muitos-sequer-teminteresse-22421007.html>. Acesso em: 26 jul. 20.

Garlet, D.; Bigolin, N. M.; Sidnei, R. S., 2016. Uma Proposta para o Ensino de Programação de Computadores na Educação Básica. Universidade Federal de Santa Maria (UFSM), Santa Maria, RS, Brasil.

Gazeta, 2016. Naiady Piva. "Inclusão da programação nos currículos escolares avança no exterior". 2016. Disponível em: $<$ https://www.gazetadopovo.com.br/educacao/inclusao-da-programacao-nos-curriculos-escolares-avanca-no-exterior9dlmbpvkpztvrp4vp164718fk/>. Acesso em: 27 jul. 20.

IDEB, 2020. Índice de Desenvolvimento da Educação Básica. Disponível em:〈http://portal.inep.gov.br/ideb〉. Acesso em: 26 jul. 20.

Morgado, José Carlos. O professor como decisor curricular: de ortodoxo a cosmopolita. Revista Tempos e Espaços em Educação, São Cristóvão, Sergipe, Brasil, v. 9, n. 18, p. 55-64, jan./abr. 2016. ISSN: 2358-1425 (versão online). Disponível em: <https://seer.ufs.br/index.php/revtee/article/view/4964>. Acesso em: 20 jul. 20.

Neto, A. de S. L.. O uso da Linguagem de Programação Voltada para as Crianças do $9^{\circ}$ ano do Ensino Fundamental do Colégio Tiradentes da Escola de Polícia. X Simpósio Linguagens e Identidades da/na Amazônia Sul-Ocidental, 2016.

PALFREY, John E GASSER, Urs. Nascidos na era digital: entendendo a primeira geração de nativos digitais. Porto Alegre: Artmed, 2011.

Ponte, J. P.; Brocardo, J.; Oliveira, H. (2006) Investigações matemáticas na sala de aula. Belo Horizonte, MG: Autêntica.

Santos, E. R. et al, 2016. Estímulo do Pensamento Computacional a partir da Computação Desplugada: uma proposta para a Educação Infantil. RELATEC - Revista Latinoamericana de Tecnología Educativa, v. 15.

UNICEF, 2020. UNICEF alerta: garantir acesso livre à internet para famílias e crianças vulneráveis é essencial na resposta à Covid-19. 2020. Disponível em: <https://www.unicef.org/brazil/comunicados-de-imprensa/unicef-alertaessencial-garantir-acesso-livre-a-internet-para-familias-e-criancas-vulneraveis>. Acesso em: 26 jul. 20.

Veja, 2017. André Fuentes. Em ranking da educação com 36 países, Brasil fica em penúltimo. 2017. Disponível em: $<$ https://veja.abril.com.br/blog/impavido-colosso/em-ranking-da-educacao-com-36-paises-brasil-fica-empenultimo/>. Acesso em: 26 jul. 20.

Wing, J. M., 2010. Computational thinking: what and why? Disponível em: http://www.cs.cmu.edu/ CompThink/resources/TheLinkWing.pdf. Acesso em: 19 jun. 20. 